\title{
Research on Open-Closed-Loop Iterative Learning Control with Variable Forgetting Factor of Mobile Robots
}

\author{
Hongbin Wang, Jian Dong, and Yueling Wang \\ Department of Electrical Engineering, Yanshan University, Qinhuangdao 066004, China \\ Correspondence should be addressed to Jian Dong; abc230206@126.com
}

Received 15 March 2016; Revised 2 August 2016; Accepted 18 August 2016

Academic Editor: Juan R. Torregrosa

Copyright ( 2016 Hongbin Wang et al. This is an open access article distributed under the Creative Commons Attribution License, which permits unrestricted use, distribution, and reproduction in any medium, provided the original work is properly cited.

\begin{abstract}
We propose an iterative learning control algorithm (ILC) that is developed using a variable forgetting factor to control a mobile robot. The proposed algorithm can be categorized as an open-closed-loop iterative learning control, which produces control instructions by using both previous and current data. However, introducing a variable forgetting factor can weaken the former control output and its variance in the control law while strengthening the robustness of the iterative learning control. If it is applied to the mobile robot, this will reduce position errors in robot trajectory tracking control effectively. In this work, we show that the proposed algorithm guarantees tracking error bound convergence to a small neighborhood of the origin under the condition of state disturbances, output measurement noises, and fluctuation of system dynamics. By using simulation, we demonstrate that the controller is effective in realizing the prefect tracking.
\end{abstract}

\section{Introduction}

Mobile robots are increasingly present in the industrial [1-3] and service robotics [4-6], particularly when motion control is becoming a hot topic in the robot field. Mobile robot depends on several controlled variables. As to the system itself, there are various complicated factors in the control process. So some good methods should be adopted in order to obtain a good effect. So far, there are many researches on the mobile robot tracking control strategy [7-9].

The nonlinear system has adopted ILC algorithm to control object [10-12], especially in the robotics control area [13, 14]. For dealing with control problem, mobile robot satisfactorily fulfills all kinds of repeatable tasks by ILC. ILC is a strict mathematics branch in the field of robotic control, which is suitable for solving nonlinear strong coupling and difficult modeling high-precision path tracking control problem. ILC does not rely on the accurate mathematical model of controlled object; its controller form is more simple and requires less prior knowledge. Therefore, trajectory tracking control of a mobile robot is a typical representative of the ILC application. Oriolo et al. [15] present an iterative learning controller that applies to mobile robots. Ostafew et al. [16] put forward a mobile robot controller that combines new ILC algorithms to reduce path-tracking errors over repeated traverses along a reference path by the experimental results in the extreme complex environments without GPS. Furgale and Barfoot [17] apply an ILC scheme on the mobile robot to achieve path following and automatic steering control in an urban area. The experimental results show that it can perform path tracking by itself. Han and Lee [18] propose an open-closed PD-type ILC algorithm to control an omnidirectional mobile robot; the proposed algorithm decreases position errors and tracks the desired path under different complex terrains effectively.

Several examples of wheeled transportation robot are given to illuminate the practical relevance of an iterative learning control approach for mobile robots. However, when the system experiences output measurement noises and state disturbances, the control methods often achieve poor effect in the practical applications. So we propose an openclosed-loop iterative learning control algorithm with variable forgetting factor in this paper. The novel ILC algorithm using the open-closed iterative learning control technique and variable forgetting factor; this algorithm is similar to Daoying and Youxian [19] but is successfully developed for mobile robot's nonlinear control system. The open-closedloop ILC algorithm is made up of two controllers: one that uses information from the previous iteration and one that 
uses information from the current iteration. In fact, previous and current available information is taken full use of, which makes all the control signals favorable to track the desired trajectory. Meanwhile, introducing variable forgetting factor, this method can filter the signal towards the direction of iteration, which can weaken the convergent influence of the system caused by state disturbances and output measurement noises. The combination will be shown to be capable of achieving high performance trajectory tracking in the simulation results, while maintaining good disturbance rejection.

The remaining parts of this paper are organized as follows. The problem is described in Section 2. The controller design and convergence analysis are given in Section 3. Simulation results are presented in detail in Section 4. The conclusion is drawn in Section 5.

\section{Problem Description}

2.1. Dynamic Models of a Mobile Robot. A typical example of a mobile robot is shown in Figure 1. The posture of the mobile robot can be described by three parameters $(x, y, \theta)$ where $x$ and $y$ are position variables and $\theta$ is a heading direction angle. It is assumed that the driving wheels of the mobile robot purely roll and do not slip. The equation is defined by

$$
\dot{x} \sin \theta-\dot{y} \cos \theta=0,
$$

where $(x, y)$ denotes the position of the center of mass. $\theta$ is the angle between $X$-axis and $X_{1}$-axis with a positive anticlockwise direction. By this formula, the kinematics of the robot can be modeled by the following differential equations:

$$
\begin{aligned}
& \dot{x}=v_{1} \cos \theta, \\
& \dot{y}=v_{1} \sin \theta, \\
& \dot{\theta}=\omega,
\end{aligned}
$$

where $v_{1}$ is the forward velocity while $\omega$ is the angular velocity of the robot. System (1) can be written in matrix as follows:

$$
\dot{q}=S(q) \nu(t),
$$

where $v(t)=\left[\begin{array}{ll}v_{1} & \omega\end{array}\right]^{T}$ and $S(q)$ is expressed as

$$
S(q)=\left[\begin{array}{cc}
\cos \theta & 0 \\
\sin \theta & 0 \\
0 & 1
\end{array}\right]
$$

with $q=(x, y, \theta)^{T}$.

2.2. Abstract Mathematical Description of the Mobile Robot System. To facilitate the proving, abstract mathematical description of the mobile robot system is represented as nonlinear system. Now we consider the nonlinear systems with state disturbances and output measurement noises for mobile robot

$$
\begin{aligned}
& \dot{x}_{k}(t)=f\left(x_{k}(t), u_{k}(t), t\right)+\beta_{k}(t), \\
& y_{k}(t)=g\left(x_{k}(t), t\right)+D(t) u_{k}(t)+\gamma_{k}(t),
\end{aligned}
$$

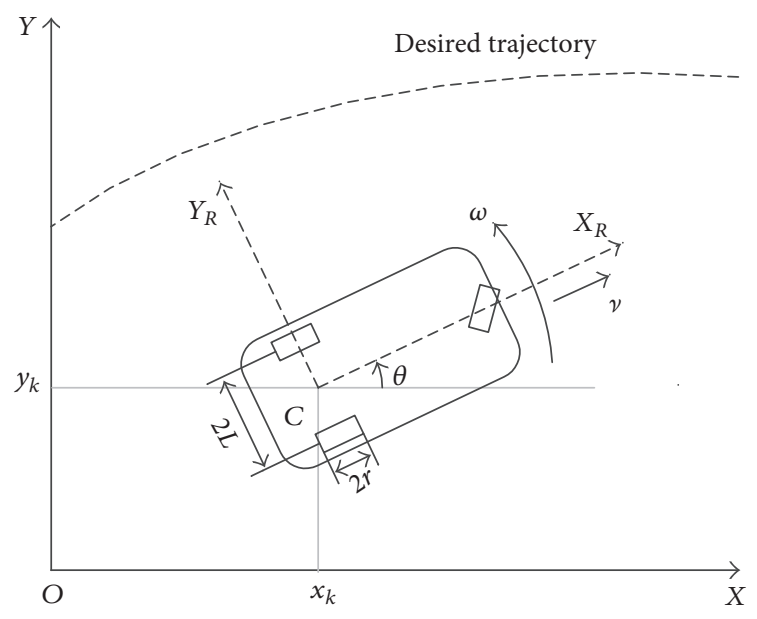

Figure 1: Configuration of mobile robot.

where $k$ and $t \in[0, T]$ are the iteration index and time, respectively. $x_{k}(t), u_{k}(t), y_{k}(t), \beta_{k}(t)$, and $\gamma_{k}(t)$ are the states, inputs, outputs, state disturbances, and output measurement noises at the $k$ th iteration. $f(\cdot), g(\cdot)$, and $D(\cdot)$ are nonlinear functions.

Assumption 1. Functions $f$ and $g$ are uniformly globally Lipschitz with respect to $x$ and $u$ on a compact set:

$$
\begin{aligned}
& \left\|f\left(x_{1}, u_{1}, t\right)-f\left(x_{2}, u_{2}, t\right)\right\| \\
& \quad \leq c_{f}\left(\left\|x_{1}-x_{2}\right\|+\left\|u_{1}-u_{2}\right\|\right), \\
& \left\|g\left(x_{1}, t\right)-g\left(x_{2}, t\right)\right\| \leq c_{g}\left(\left\|x_{1}-x_{2}\right\|\right),
\end{aligned}
$$

where $c_{f}$ and $c_{g}$ are the Lipschitz constants.

Assumption 2. Initial error of the system at the $k$ th trial satisfying this criterion

$$
\left\|x_{k+1}(0)-x_{k}(0)\right\| \leq b_{x_{0}}, \quad b_{x_{0}}>0, \forall k .
$$

Bound Assumptions. The desired input, the system disturbance, and the measurement noise are bounded, such that, for all $k$,

$$
\begin{array}{r}
\max _{0 \leq t \leq T}\|D(t)\| \leq c_{d}, \\
\max _{0 \leq t \leq T}\left\|u_{d}(t)\right\| \leq b_{u_{d}}, \\
\max _{1 \leq k \leq \infty} \max _{0 \leq t \leq T}\left\|\beta_{k}(t)\right\| \leq b_{\beta}, \\
\max _{1 \leq k \leq \infty} \max _{0 \leq t \leq T}\left\|\gamma_{k}(t)\right\| \leq b_{\gamma},
\end{array}
$$

where $c_{d}, b_{u_{d}}, b_{\beta}$, and $b_{\gamma}$ are the positive constants.

\section{Controller Design and Convergence Analysis}

To make further improvement on the tracking precision of system, we will find an ideal input by the method of iterative 
learning. And it can make the output of system close to the desired trajectory as far as possible.

In the work, the forgetting factor $a$ is introduced to the designed controller. The factor $a$ is used to make a tradeoff between perfect learning and robustness, which can increase the robustness of ILC against uncertainty, state disturbances, and output measurement noises and fluctuation of system dynamics.

Now we propose an open-closed-loop iterative learning control with variable forgetting factor as

$$
\begin{aligned}
u_{k+1}(t)= & a u_{0}(t)+(1-a) u_{k}(t)+L_{1}(t) e_{k}(t) \\
& +L_{2}(t) e_{k+1}(t)
\end{aligned}
$$

where $k$ indicates the iteration number, $e_{k}=y_{d}-y_{k}$ are the output tracking errors, and $L_{1}(t)$ and $L_{2}(t)$ are the learning gain matrices that satisfy $L_{1}(t) \leq c_{L_{1}}, L_{2}(t) \leq c_{L_{2}} \cdot a$ is variable forgetting factor, and $u_{0}(t)$ is the initial value of input.

The tracking ability of the system is stronger for smaller values of the forgetting factor $a$, and vice versa. We generally use the fixed forgetting factor, but $a$ cannot vary with the change of the system features. Therefore, variable forgetting factor $a$ is introduced in this paper, which can vary automatically according to changing system's deviation:

$$
\begin{aligned}
& a=(1-\beta) 50^{L}, \\
& L=-\max \left[r e_{k}^{2}(t)\right],
\end{aligned}
$$

where $r$ is sensitive to gain; $r$ is used for controlling the rate in which $\beta$ approaches 1 . If $r$ is small, $L$ becomes large, and the convergence speed is reduced. If $r$ is large, the system stability decreases.

Let $\Delta x_{k}=x_{d}-x_{k}, e_{k}=y_{d}-y_{k}$, and $\Delta u_{k}=u_{d}-u_{k}$.

From (1), we get

$$
\begin{aligned}
\left\|\Delta x_{k+1}\right\|=\left\|x_{d}-x_{k+1}\right\| \\
=\left\|\int_{0}^{t}\left[f\left(x_{d}, u_{d}, s\right)-f\left(x_{k+1}, u_{k+1}, s\right)-\beta_{k}(t)\right] d s\right\| .
\end{aligned}
$$

By the Lipschitz condition, we have

$$
\begin{aligned}
& \left\|\Delta x_{k+1}\right\| \\
& \leq c_{f} \int_{0}^{t}\left[\left\|x_{d}(s)-x_{k+1}(s)\right\|+\left\|u_{d}(s)-u_{k+1}(s)\right\|\right] d s \\
& \quad+b_{\beta} .
\end{aligned}
$$

Applying the Bellman-Gronwall theorem to (12) gives

$$
\begin{aligned}
\left\|\Delta x_{k+1}\right\| \leq & c_{f} \int_{0}^{t} e^{c_{f}(t-s)} \int_{0}^{s}\left\|u_{d}(\tau)-u_{k+1}(\tau)\right\| d \tau d s \\
& +b_{\beta} \\
= & c_{f} \int_{0}^{t} e^{c_{f}(t-s)} \int_{0}^{s}\left\|\Delta u_{k+1}(\tau)\right\| d \tau d s+b_{\beta} .
\end{aligned}
$$

Similarly, multiplying both sides of (13) by $e^{-\lambda t}$ and taking $\lambda$-norm yield

$$
\begin{gathered}
\left\|\Delta x_{k+1}\right\|_{\lambda} \leq\left(\frac{c_{f}-1}{\lambda} e^{c_{f} T}-c_{f} e^{\left(c_{f}-\lambda\right) T}\right)\left\|\Delta u_{k+1}\right\|_{\lambda}+b_{\beta} . \\
\text { Let } w=\left(\left(c_{f}-1\right) / \lambda\right) e^{c_{f} T}-c_{f} e^{\left(c_{f}-\lambda\right) T} ; \text { we have } \\
\left\|\Delta x_{k+1}\right\|_{\lambda} \leq w\left\|\Delta u_{k+1}\right\|_{\lambda}+b_{\beta} .
\end{gathered}
$$

Inserting $\Delta u_{k}=u_{d}-u_{k}$ into $\Delta u_{k+1}=u_{d}-u_{k+1}$ gives

$$
\Delta u_{k+1}=\Delta u_{k}-u_{k+1}+u_{k}
$$

On the other hand, it follows from (9) that the control input becomes

$$
\begin{aligned}
\Delta u_{k+1} & =\Delta u_{k}-u_{k+1}+u_{k} \\
& =\Delta u_{k}-a u_{0}+a u_{k}-L_{1} e_{k}-L_{2} e_{k+1} \\
& =\Delta u_{k}-a u_{0}+a\left(u_{d}-\Delta u_{k}\right)-L_{1} e_{k}-L_{2} e_{k+1} \\
& =(I-a) \Delta u_{k}-L_{1} e_{k}-L_{2} e_{k+1}+\left(a u_{d}-a u_{0}\right) .
\end{aligned}
$$

From (5), we have

$$
\begin{aligned}
e_{k} & =y_{d}-y_{k} \\
& =g\left(x_{d}, t\right)+D u_{d}-g\left(x_{k}, t\right)-D u_{k}-\gamma_{k}, \\
e_{k+1} & =g\left(x_{d}, t\right)+D u_{d}-g\left(x_{k+1}, t\right)-D u_{k+1}-\gamma_{k+1} .
\end{aligned}
$$

Substituting (18) into (17) gives

$$
\begin{aligned}
& \Delta u_{k+1}=(I-a) \Delta u_{k}-L_{1} e_{k}-L_{2} e_{k+1}+\left(a u_{d}-a u_{0}\right) \\
& =(I-a) \Delta u_{k}-L_{1}\left[g\left(x_{d}, t\right)+D u_{d}-g\left(x_{k}, t\right)\right. \\
& \left.-D u_{k}-\gamma_{k}\right]-L_{2}\left[g\left(x_{d}, t\right)+D u_{d}-g\left(x_{k+1}, t\right)\right. \\
& \left.-D u_{k+1}-\gamma_{k+1}\right]+\left(a u_{d}-a u_{0}\right)=(I-a) \Delta u_{k} \\
& -L_{1}\left[g\left(x_{d}, t\right)-g\left(x_{k}, t\right)+D \Delta u_{k}-\gamma_{k}\right] \\
& -L_{2}\left[g\left(x_{d}, t\right)-g\left(x_{k+1}, t\right)+D \Delta u_{k+1}-\gamma_{k+1}\right] \\
& +\left(a u_{d}-a u_{0}\right)=\left(I-a-L_{1} D\right) \Delta u_{k}-L_{1}\left[g\left(x_{d}, t\right)\right. \\
& \left.-g\left(x_{k}, t\right)\right]-L_{2}\left[g\left(x_{d}, t\right)-g\left(x_{k+1}, t\right)\right] \\
& -L_{2} D \Delta u_{k+1}+\left(a u_{d}-a u_{0}+L_{1} \gamma_{k}+L_{2} \gamma_{k+1}\right) .
\end{aligned}
$$

Let us take norm on both sides of (19). Using Lipschitz condition gives

$$
\begin{aligned}
\left\|\Delta u_{k+1}\right\| \leq & \left\|I-a-L_{1} D\right\|\left\|\Delta u_{k}\right\|+c_{L_{1}} c_{g}\left\|x_{d}-x_{k}\right\| \\
& +c_{L_{2}} c_{g}\left\|x_{d}-x_{k+1}\right\|+c_{L_{2}} c_{d}\left\|\Delta u_{k+1}\right\| \\
& +\left[a b_{u_{d}}+a u_{0}+\left(L_{1}+L_{2}\right) b_{\gamma}\right] \\
\leq & \left\|I-a-L_{1} D\right\|\left\|\Delta u_{k}\right\|+c_{L_{1}} c_{g}\left\|\Delta x_{k}\right\| \\
& +c_{L_{2}} c_{g}\left\|\Delta x_{k+1}\right\|+c_{L_{2}} c_{d}\left\|\Delta u_{k+1}\right\|+\xi
\end{aligned}
$$

where $\xi=a b_{u_{d}}-a u_{0}+\left(L_{1}+L_{2}\right) b_{\gamma}$. 
Multiplying both sides of (20) by $e^{-\lambda t}$ to compute the $\lambda$ norm, we have

$$
\begin{aligned}
\left\|\Delta u_{k+1}\right\|_{\lambda} \leq & \left\|I-a-L_{1} D\right\|\left\|\Delta u_{k}\right\|_{\lambda}+c_{L_{1}} c_{g}\left\|\Delta x_{k}\right\|_{\lambda} \\
& +c_{L_{2}} c_{g}\left\|\Delta x_{k+1}\right\|_{\lambda}+c_{L_{2}} c_{d}\left\|\Delta u_{k+1}\right\|_{\lambda}+\xi .
\end{aligned}
$$

Inserting (15) into (21) gives

$$
\begin{aligned}
\left\|\Delta u_{k+1}\right\|_{\lambda} \leq & \left\|I-a-L_{1} D\right\|\left\|\Delta u_{k}\right\|_{\lambda} \\
& +c_{L_{1}} c_{g}\left(w\left\|\Delta u_{k}\right\|_{\lambda}+b_{\beta}\right) \\
& +c_{L_{2}} c_{g}\left(w\left\|\Delta u_{k+1}\right\|_{\lambda}+b_{\beta}\right) \\
& +c_{L_{2}} c_{d}\left\|\Delta u_{k+1}\right\|_{\lambda}+\xi \\
\leq & \left\|I-a-L_{1} D\right\|\left\|\Delta u_{k}\right\|_{\lambda}+c_{L_{1}} c_{g} w\left\|\Delta u_{k}\right\|_{\lambda} \\
& +c_{L_{2}} c_{g} w\left\|\Delta u_{k+1}\right\|_{\lambda}+c_{L_{2}} c_{d}\left\|\Delta u_{k+1}\right\|_{\lambda}+\xi \\
& +\left(c_{L_{1}} c_{g}+c_{L_{2}} c_{g}\right) b_{\beta} .
\end{aligned}
$$

Then (22) implies

$$
\left\|\Delta u_{k+1}\right\|_{\lambda} \leq \rho\left\|\Delta u_{k}\right\|_{\lambda}+\varepsilon
$$

where $\rho=\left(\left\|I-a-L_{1} D\right\|+c_{L_{1}} c_{g} w\right) /\left(1-c_{L_{2}} c_{g} w-c_{L_{2}} c_{d}\right)$ and $\varepsilon=\left(\xi+\left(c_{L_{1}} c_{g}+c_{L_{2}} c_{g}\right) b_{\beta}\right) /\left(1-c_{L_{2}} c_{g} w-c_{L_{2}} c_{d}\right)$.

Let us choose suitable parameters $\lambda, L_{1}$, and $L_{2}$ so that $\rho<$ 1. According to limit definition, we can reach the conclusion $\lim _{k \rightarrow \infty}\left\|\Delta u_{k}\right\|_{\lambda} \leq \varepsilon^{*}, u_{k} \rightarrow u_{d}$. $y_{d}$.

Similarly, when $k \rightarrow \infty, x_{k} \rightarrow x_{d}$. When $k \rightarrow \infty, y_{k} \rightarrow$

\section{Simulation}

To show that the proposed ILC scheme is feasible, we consider the mobile robot as a simulation. The desired trajectory is given.

$$
\begin{aligned}
& x(t)=\cos t, \\
& y(t)=\sin t, \\
& \theta(t)=t+\frac{\pi}{2} .
\end{aligned}
$$

The initial states are $u_{0}(0)=0$ and $q_{k}(0)=$ $(0,0,0)^{T}$. The interference terms are $\beta_{k}(t)=$ $0.01[\sin (40 \pi t) \sin (40 \pi t) \quad 0.5 \sin (40 \pi t)]^{T}$.

We choose an open-closed-loop iterative learning control algorithm with variable forgetting factor:

$$
\begin{aligned}
u_{k+1}(t)= & a u_{0}(t)+(1-a) u_{k}(t)+L_{1}(t) e_{k}(t) \\
& +L_{2}(t) e_{k+1}(t) .
\end{aligned}
$$

The data used in the simulation is chosen as

$$
\begin{aligned}
\beta & =0.9, \\
r & =5, \\
u_{0} & =\left[\begin{array}{l}
0.1 \\
0.1
\end{array}\right],
\end{aligned}
$$

$$
L_{1}(t)=L_{2}(t)=0.3\left[\begin{array}{ccc}
\cos t & \sin t & 0 \\
0 & 0 & 1
\end{array}\right] \text {. }
$$

After checking the convergence condition, we can obtain

$\rho$

$$
\begin{aligned}
& =\frac{\left[\left\|I-a-L_{1} D\right\|+c_{L_{1}} c_{g}\left(\left(\left(c_{f}-1\right) / \lambda\right) e^{c_{f} T}-c_{f} e^{\left(c_{f}-\lambda\right) T}\right)\right]}{1-c_{L_{2}} c_{g}\left(\left(\left(c_{f}-1\right) / \lambda\right) e^{c_{f} T}-c_{f} e^{\left(c_{f}-\lambda\right) T}\right)-c_{L_{2}} c_{d}} \\
& =0.72<1 .
\end{aligned}
$$

According to limit definition, we can reach the conclusion of this theorem: $\lim _{k \rightarrow \infty}\left\|\Delta u_{k}\right\|_{\lambda} \leq \varepsilon^{*}, u_{k} \rightarrow u_{d}$. Similarly, when $k \rightarrow \infty, x_{k} \rightarrow x_{d}$. When $k \rightarrow \infty, y_{k} \rightarrow y_{d}$.

Simulation results are shown in Figures 2, 3, and 4.

To compare the performance of the proposed ILC algorithm with that of conventional ILC algorithm, we carry out the simulation in the same conditions and parameters. From Figure 2 we can see that the new ILC algorithm performs better in tracking performance. Meanwhile, we find that the proposed ILC algorithm has smaller error after 20 iterations and faster convergence speed, as shown in Figure 3. But, for the traditional ILC algorithm without variable forgetting factor, there are large tracking errors. Figure 4 shows that the proposed ILC algorithm has smaller tracking errors than the ILC algorithm without variable forgetting factor in the last iteration. Large errors are not allowable for engineering applications.

Therefore, from the simulation results, we can draw a conclusion that the proposed ILC algorithm has good performance in tracking desired trajectory and the robustness against output measurement noises and state and external disturbances, even though there are some small steadystate errors, which may be neglected in practice. Most of the achievements are attributed to variable forgetting factor. Because of the existence of initial error, state disturbances, and output measurement noises, the tracking error cannot approach zero. If these disturbances, noises, and initial errors are not present, then the input, state, and output errors converge to zero.

\section{Conclusions}

To a class of nonlinear mobile robot system, a novel ILC algorithm is proposed. By introduction of variable forgetting factor, this method weakens the convergent influence of the system caused by state disturbances and output measurement noises. The update law exploits both predictive and current learning terms to enhance stability characteristics and drives the inputs, states, and outputs to their desired ones within 

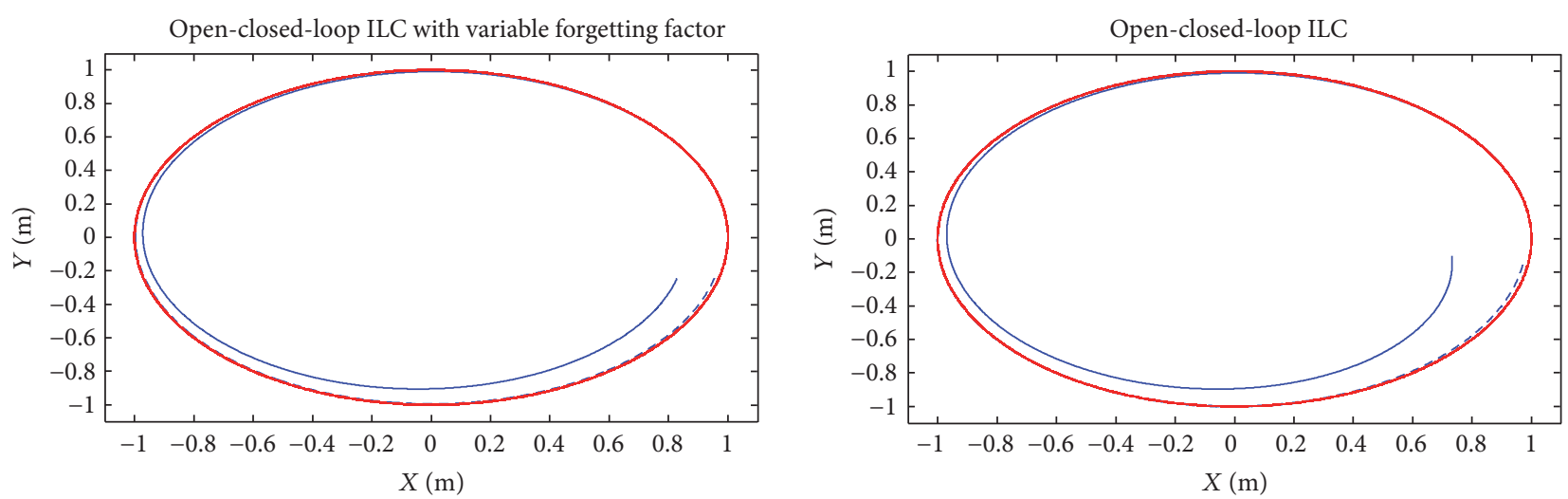
$-k=20$
-..-k $=200$
— Desired trajectory
$k=20$
-.. $k=200$
— Desired trajectory
$---k=40$
$---k=40$

FIgURE 2: Desired and actual position trajectories.
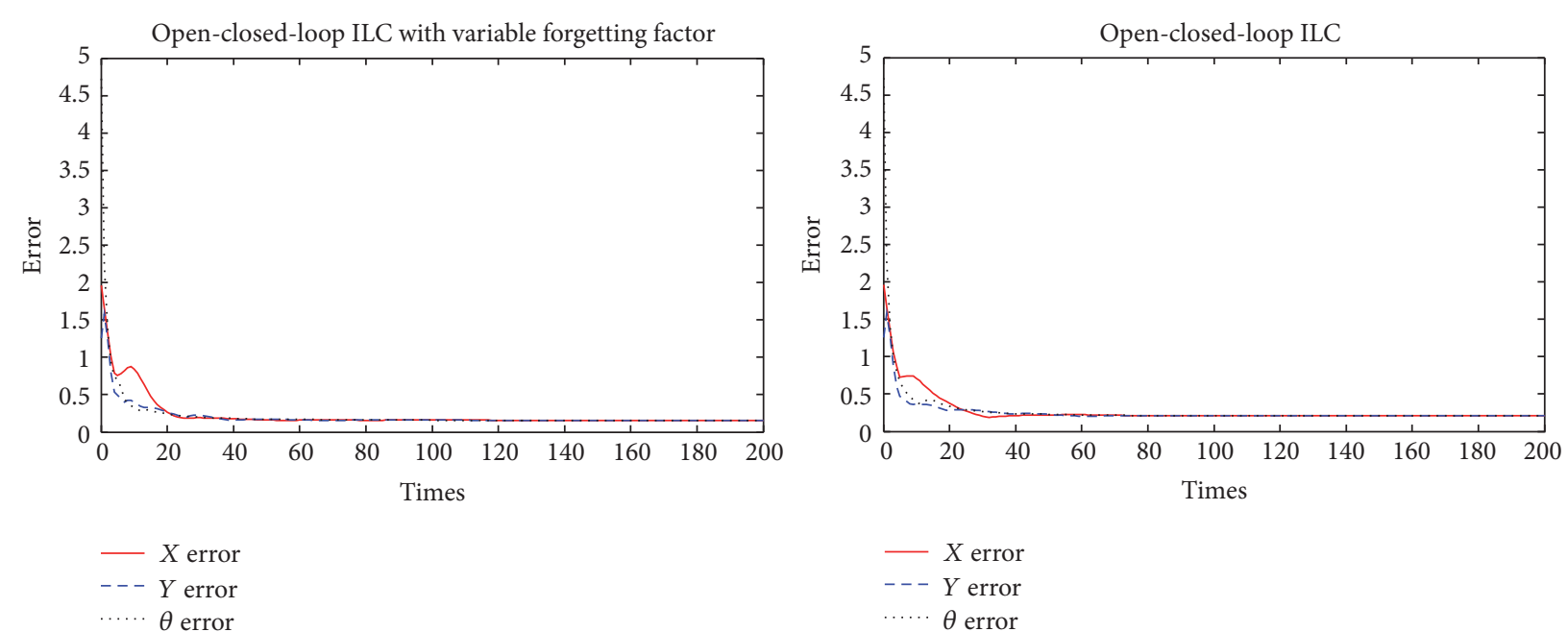

FIgURE 3: Max absolute values of the tracking error.
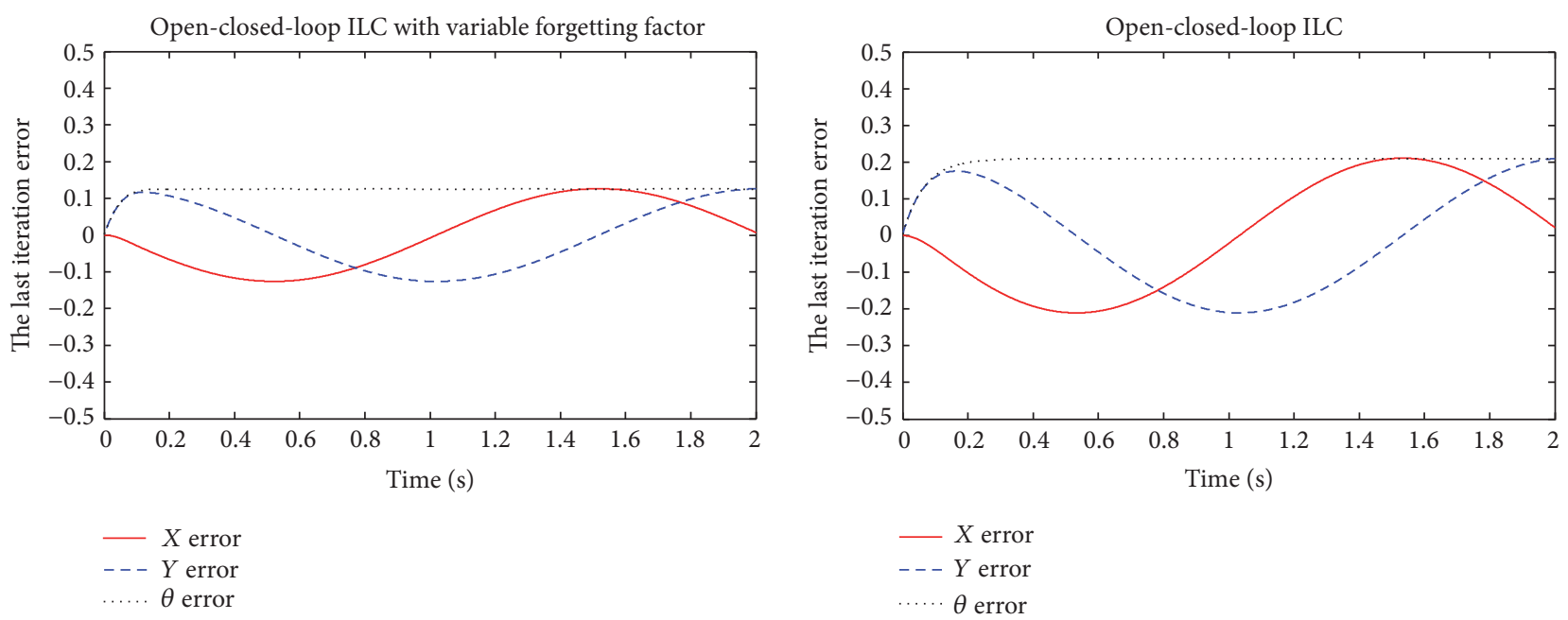

FIgURE 4: Tracking error in the last iteration. 
bounds. The results of simulation show that the system has obtained excellent trajectory tracking effect. Our future work is extending the results to other uncertain dynamics of the mobile robot.

\section{Competing Interests}

The authors declare that there is not conflict of interests regarding the publication of this paper.

\section{Acknowledgments}

This work is supported by the National Natural Science Foundation of China (Grant no. 61473248) and Natural Science Foundation of Hebei Province of China (Grant no. F2016203496).

\section{References}

[1] Q. Tang, "Localization and tracking control for mobile welding robot," Industrial Robot, vol. 41, no. 3, pp. 259-265, 2014.

[2] P. J. Navarro, C. Fernandez, and P. Sanchez, "Industriallike vehicle platforms for postgraduate laboratory courses on robotics," IEEE Transactions on Education, vol. 56, no. 1, pp. 3441, 2013.

[3] S. B. Saoud, L. Nciri, and M. Ghrissi, "Path-tracking and parking manoeuvre control of an industrial tricycle robot," International Journal of Robotics and Automation, vol. 20, no. 4, pp. 231-238, 2005.

[4] H. Wu, G.-H. Tian, Y. Li, F.-Y. Zhou, and P. Duan, "Spatial semantic hybrid map building and application of mobile service robot," Robotics and Autonomous Systems, vol. 62, no. 6, pp. 923941, 2014.

[5] C.-C. Tsai, H.-C. Huang, and S.-C. Lin, "FPGA-based parallel DNA algorithm for optimal configurations of an omnidirectional mobile service robot performing fire extinguishment," IEEE Transactions on Industrial Electronics, vol. 58, no. 3, pp. 1016-1026, 2011.

[6] Y. Zhuang, B. Li, Y. Liu, and H. Liu, "Fusing monocular vision and laser range data for human detection and tracking via multi-robot collaboration," Sensor Letters, vol. 10, no. 8, pp. 1607-1620, 2012.

[7] M. Szuster and Z. Hendzel, "Discrete globalised dual heuristic dynamic programming in control of the two-wheeled mobile robot," Mathematical Problems in Engineering, vol. 2014, Article ID 628798, 16 pages, 2014.

[8] L. Zhang, C.-C. Lim, Y. Chen, and H. R. Karimi, "Tracking mobile robot in indoor wireless sensor networks," Mathematical Problems in Engineering, vol. 2014, Article ID 837050, 8 pages, 2014.

[9] C. Jung, C.-B. Moon, D. Jung, J. Choi, and W. Chung, "Design of test track for accurate calibration of two wheel differential mobile robots," International Journal of Precision Engineering and Manufacturing, vol. 15, no. 1, pp. 53-61, 2014.

[10] J.-M. Wei, Y.-A. Hu, and M.-M. Sun, "Observer-based adaptive iterative learning control for a class of nonlinear time delay systems with input saturation," Mathematical Problems in Engineering, vol. 2015, Article ID 645161, 19 pages, 2015.

[11] G. Xu, C. Shao, Y. Han, and K. Yim, "New quasi-Newton iterative learning control scheme based on rank-one update for nonlinear systems," The Journal of Supercomputing, vol. 67, no. 3, pp. 653-670, 2014.

[12] X. Bu, F. Yu, Z. Hou, and F. Wang, "Iterative learning control for a class of nonlinear systems with random packet losses," Nonlinear Analysis: Real World Applications, vol. 14, no. 1, pp. 567-580, 2013.

[13] F. Bouakrif, D. Boukhetala, and F. Boudjema, "Velocity observer-based iterative learning control for robot manipulators," International Journal of Systems Science: The Theory and Practice of Mathematical Modelling, Simulation, Optimization and Control in Relation to Biological, Economic, Industrial and Transportation Systems, vol. 44, no. 1, pp. 214-222, 2013.

[14] H. Chen, G. Xing, and H. Sun, "Indirect iterative learning control for robot manipulator with non-Gaussian disturbances," IET Control Theory \& Applications, vol. 7, no. 17, pp. 2090-2102, 2013.

[15] G. Oriolo, S. Panzieri, and G. Ulivi, "An iterative learning controller for nonholonomic mobile robots," International Journal of Robotics Research, vol. 17, no. 9, pp. 954-970, 1998.

[16] C. J. Ostafew, A. P. Schoellig, and T. D. Barfoot, "Visual teach and repeat, repeat, repeat: Iterative Learning Control to improve mobile robot path tracking in challenging outdoor environments," in Proceedings of the IEEE/RSJ International Conference on Intelligent Robots and Systems, pp. 176-181, Tokyo, Japan, November 2013.

[17] P. Furgale and T. D. Barfoot, "Visual teach and repeat for longrange rover autonomy, Journal of Field Robotics, vol. 27, no. 5, pp. 534-560, 2010.

[18] K.-L. Han and J. S. Lee, "Iterative path tracking of an omnidirectional mobile robot," Advanced Robotics, vol. 25, no. 13-14, pp. 1817-1838, 2011.

[19] P. Daoying and S. Youxian, "A preliminary survey on the progress and future research directions of open-closed-loop iterative learning control," Mechanical \& Electrical Engineering Magazine, vol. 5, no. 8, pp. 65-72, 1999. 


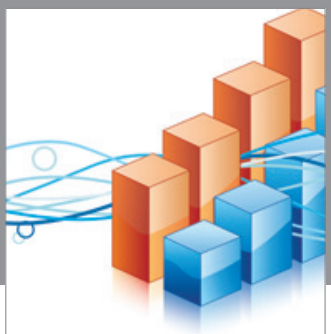

Advances in

Operations Research

vatem alat4

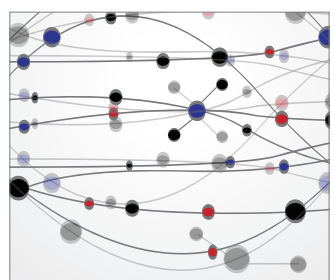

\section{The Scientific} World Journal
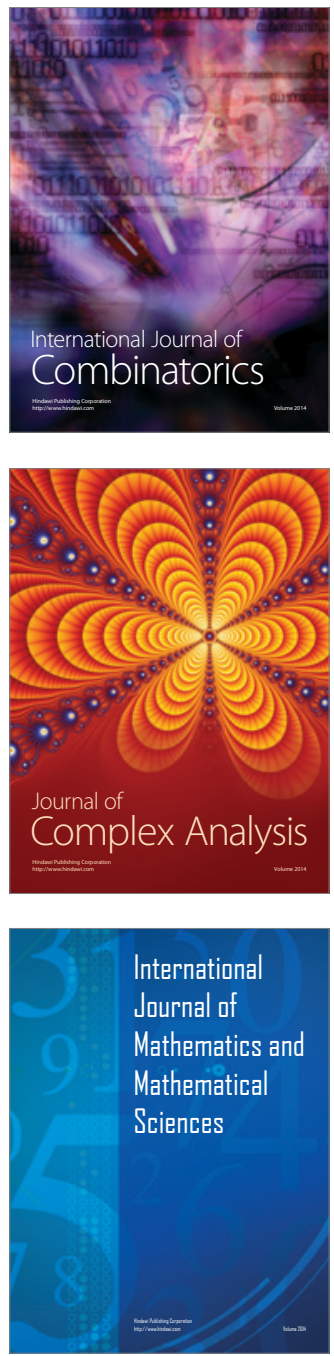
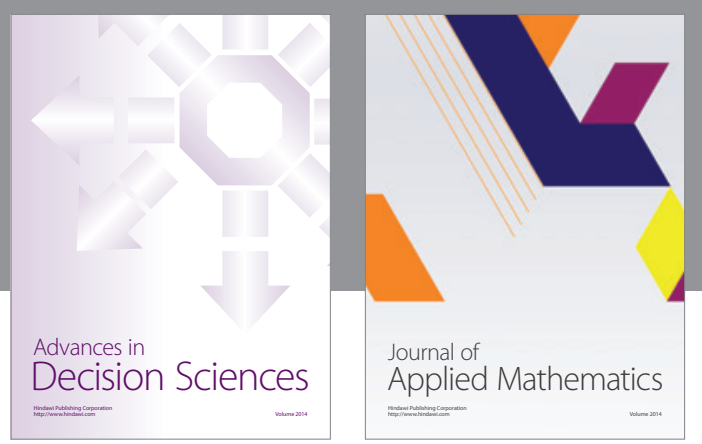

Algebra

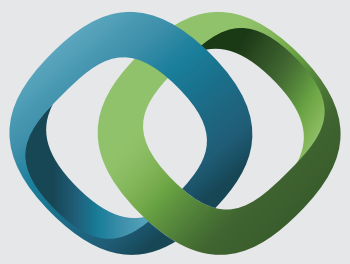

\section{Hindawi}

Submit your manuscripts at

http://www.hindawi.com
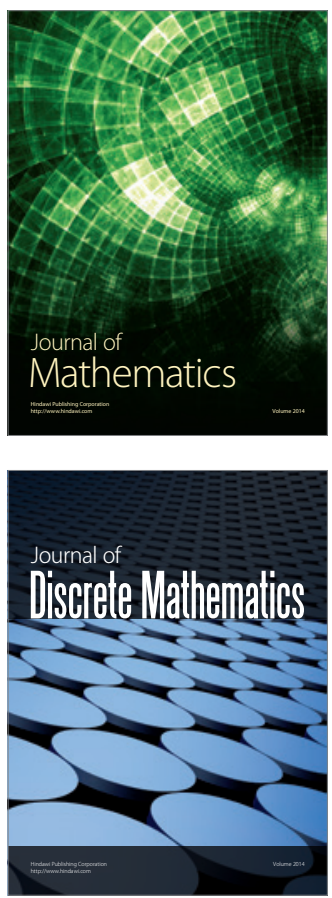

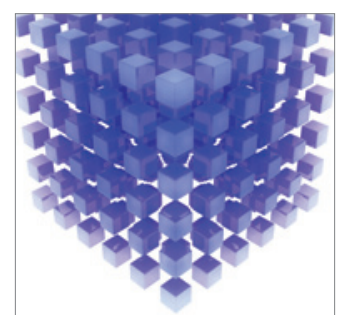

Mathematical Problems in Engineering
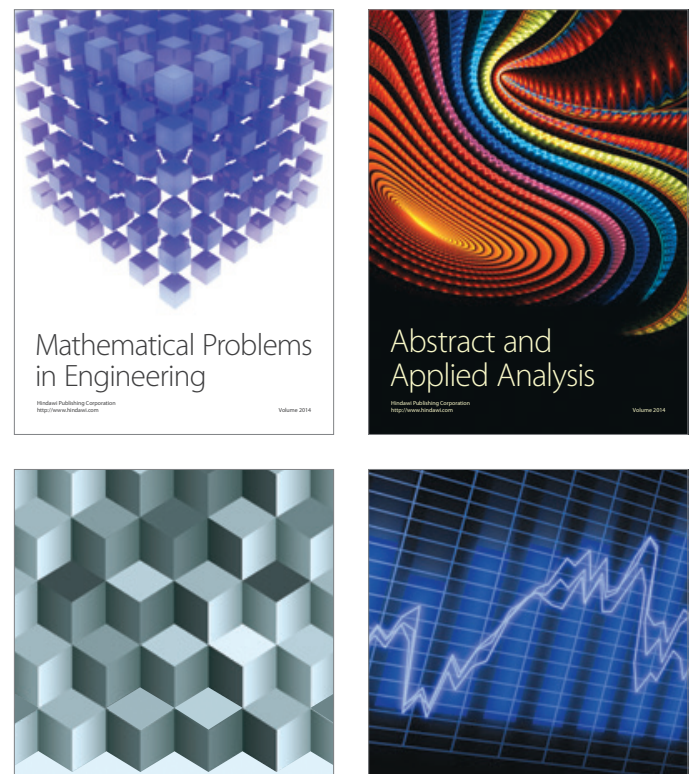

Journal of

Function Spaces

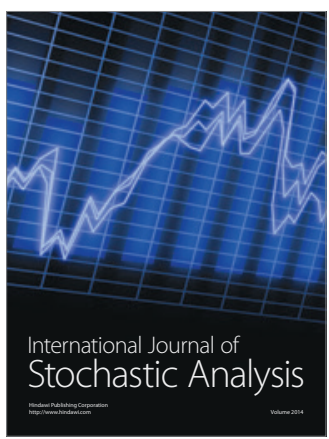

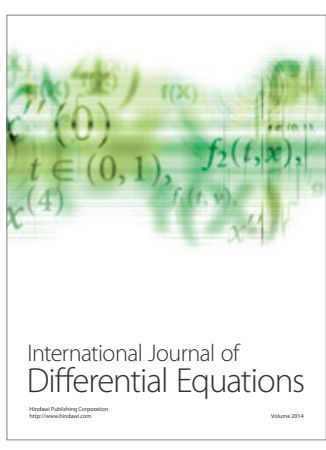
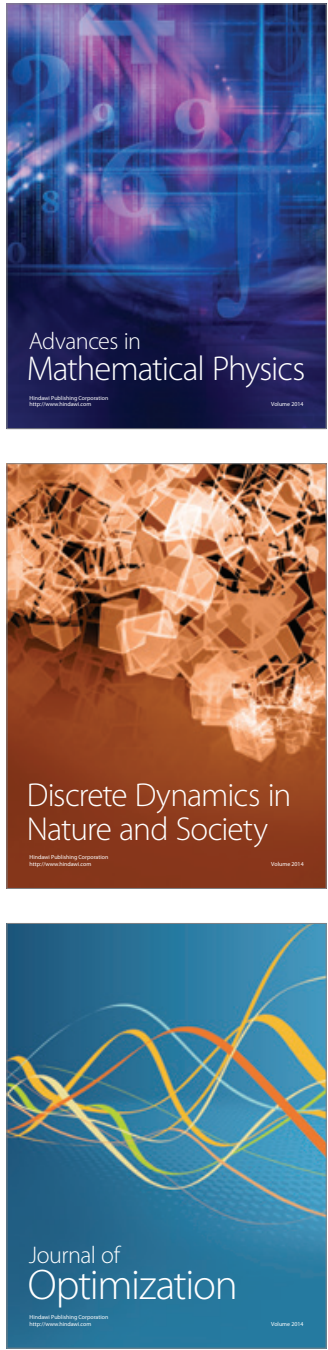